\begin{tabular}{|l|c|c|c|r|}
\hline $\begin{array}{l}\text { Cuadernos de Investigación Geográfica } \\
\text { Geographical Research Letters }\end{array}$ & 2017 & N $^{\circ} 43(1)$ & pp. 329-349 & $\begin{array}{r}\text { ISSN 0211-6820 } \\
\text { eISSN 1697-9540 }\end{array}$ \\
\hline
\end{tabular}

\title{
FRAGMENTACIÓN DE LA RED NATURA 2000 POR INFRAESTRUCTURAS VIARIAS DE TRANSPORTE EN MALLORCA
}

\author{
R. ROSSELLÓ-MELIS, J. LORENZO-LACRUZ* \\ ${ }^{1}$ Department of Geography, University of the Balearic Islands. Ctra. de Valldemossa km 7.5, \\ 07122 Palma de Mallorca, Balearic Islands, Spain.
}

RESUMEN. La fragmentación de hábitats está considerada como una de las principales causas de la actual crisis de biodiversidad. Particularmente, la presencia de infraestructuras lineales de transporte representa una de las principales causas de la fragmentación de hábitats y sus efectos suponen una amenaza para la diversidad y riqueza de los hábitats y para la capacidad de dispersión de las especies. En este estudio se realiza una evaluación del estado de fragmentación de los hábitats de la Red Natura 2000 (LIC y ZEPA) de Mallorca (Islas Baleares) y se analiza su relación con las infraestructuras viarias de transporte, a partir de la aplicación de distintos indicadores fisiográficos y morfométricos. En líneas generales, los análisis realizados muestran un mayor grado de fragmentación de los hábitats Natura 2000 del norte de la isla y de la parte occidental de la Sierra de Tramuntana. Estos últimos también presentan una mayor vulnerabilidad ante las perturbaciones externas, con una mayor proporción de hábitat de borde respecto al hábitat interior. En cuanto a los impactos causados por las carreteras, si bien la media de uso de las infraestructuras que atraviesan espacios mallorquines protegidos se sitúa en 5500 vehículos/día, las mayores afecciones por tráfico rodado se concentran en determinados tramos de la sierra de Tramuntana y en el norte de la isla (bahía de Pollença, bahía de Alcudia y Artá), donde la intensidad de uso supera los 10.000 vehículos diarios.

Natura 2000 network fragmentation caused by road infrastructures in Mallorca

ABSTRACT. Habitat fragmentation is considered a main cause of the global biodiversity crisis. Particularly, the presence of linear transport infrastructures is one of the main causes of habitat fragmentation and its effects represent a threat to the diversity and richness of habitats and to the dispersal capacity of species. This study assesses the state of fragmentation of Natura 2000 habitats (LIC and ZEPA) on Mallorca (Balearic Islands) and analyses its relationship with the presence of road infrastructures, through application of different physiographic and morphometric indicators. The analysis shows a higher degree 
of fragmentation in the Natura 2000 habitats located in north of Mallorca and the western sector of the Tramuntana mountain range. Those are also more vulnerable to external disturbance, due to a higher proportion of edge habitat in relation to interior habitat. The average use of roads that intersect with protected areas is 5500 vehicles per day. However, the main affectations caused by traffic are concentrated in specific sectors of the Tramuntana range and in the north of the island (Pollença bay, Alcúdia bay and Artá) where the average use is higher than 10.000 vehicles per day.

Palabras clave: fragmentación, conservación de la biodiversidad, Sistemas de Información Geográfica, región Mediterránea.

Key words: fragmentation, biodiversity conservation, Geographic Information Systems, Mediterranean region.

Recibido: 18 December 2016

Aceptado: 21 March 2017

* Correspondencia: Jorge Lorenzo-Lacruz, Department of Geography, University of Balearic Islands, 07122 Palma de Mallorca, Spain. E-mail: j.lorenzo@uib.es

\section{Introducción}

Históricamente, las relaciones entre las sociedades humanas y el medio natural han provocado una progresiva transformación del territorio que se ha intensificado en las últimas décadas debido a un incremento de la población, la sobreexplotación de recursos y una capacidad tecnológica sin precedentes (Santos y Tellería, 2006), las cuales han ocasionado repercusiones significativas en la biodiversidad. La magnitud de estas transformaciones, especialmente las ocurridas durante el siglo $\mathrm{XX}$, compromete la conservación de los ecosistemas y organismos que los habitan (Ministerio de Medio Ambiente y Medio Rural y Marino, 2010). Un factor clave de transformación del territorio es la fragmentación de hábitats, la cual es considerada por numerosos autores como una de las principales causas de la crisis global de biodiversidad en la que estamos inmersos y una de las mayores amenazas para la conservación de hábitats y ecosistemas singulares (Santos y Tellería, 2006; Gurrutxaga y Lozano, 2010; García, 2011). Fundamentalmente, la fragmentación disminuye el territorio disponible para los hábitats, lo que provoca a su vez una creciente atomización de sus poblaciones faunísticas (Santos y Tellería, 2006). El concepto de fragmentación de hábitats varía en función del enfoque epistemológico del autor. Lindenmayer y Fischer (2006), definen la fragmentación como un cambio en la estructura y configuración de los hábitats naturales dentro de un paisaje, mientras que Hargis et al. (1999), Myers et al. (2000), Múgica de la Guerra et al. (2002) o Brook et al. (2003) sostienen que la fragmentación se refiere principalmente a la pérdida y degradación de hábitats, ya que un fragmento del paisaje es transformado para un diferente tipo de uso de la tierra. 
Desde una perspectiva morfológica-espacial, la fragmentación conlleva la transformación de los ecosistemas y hábitats en un conjunto de parches aislados (fragmentos), rodeados de otros hábitats, distintos al original, denominados matriz (García, 2011). Esta alteración de hábitats o ecosistemas se traduce en un cambio progresivo en el paisaje, haciendo visible una pérdida de superficie de hábitat original, un aumento de los fragmentos de matriz y un aumento de la relación perímetro/superficie, lo que origina un incremento del efecto borde (Gurrutxaga y Lozano, 2006; Martín et al., 2006; Santos y Tellería, 2006; Ministerio de Medio Ambiente y Medio Rural y Marino, 2010; Jaeger et al., 2011; García, 2011; Szek, 2012).

Es importante destacar la importancia del dinamismo temporal del proceso de modificación del paisaje (Szek, 2012), ya que los efectos de la fragmentación no son lineales, sino que se multiplican al alcanzar un porcentaje de hábitat destruido (Santos y Tellería, 2006). Las causas que explican la fragmentación de los hábitats son diversas, aunque la intensificación de la agricultura y el aprovechamiento forestal, la expansión urbanística y los procesos de industrialización, y la construcción de infraestructuras de transporte emergen como las principales (EUROPARC-España, 2009; Gurrutxaga y Lozano, 2010).

La reciente proliferación bibliográfica sobre los efectos de la fragmentación de hábitats ha obligado a una revisión de los conceptos. El Ministerio de Medio Ambiente y Medio Rural y Marino (2010) detalla distintos tipos de efectos que las infraestructuras lineales de transporte ejercen sobre los hábitats en términos de fragmentación, entre los que destacan: i) destrucción de hábitats; ii) disminución del tamaño de los hábitats: la implantación de infraestructuras lineales de transporte provoca una división de los hábitats en fragmentos más pequeños que, inevitablemente, supone una menor capacidad de albergar individuos (MacArthur y Wilson, 1967); iii) efecto borde: asociado a infraestructuras lineales de transporte que produce perturbaciones diversas como difusión de contaminantes, tanto atmosféricos como sólidos, ruido generado por el tráfico rodado, contaminación lumínica procedentes de las fuentes de luz fija y de los propios vehículos, y frecuentación antrópica como consecuencia del aumento de accesibilidad implícita que aporta la infraestructura; iv) procesos del margen de las infraestructuras que dan lugar a efectos biológicos específicos como la creación de nuevos hábitats, la canalización de la dispersión de los organismos o la canalización de los flujos de agua y sedimentos; v) las infraestructuras restringen la dispersión natural de las especies dependiendo de las características físicas de la vía y de las pautas de comportamiento de la especie (Mader, 1984; Gurrutxaga y Lozano, 2010); vi) mortalidad por atropello: las colisiones de vehículos con animales supone un problema de seguridad vial creciente que depende de las características de la vía, la intensidad de tráfico, la abundancia de organismos y el espacio por donde transcurre la infraestructura.

La complejidad e interrelación de los elementos de los sistemas naturales provoca que cualquier alteración denote cambios drásticos y frecuentemente irreversibles en el medio (Szek, 2012). Según indica el Ministerio de Medio Ambiente y Medio Rural y Marino (2010), la fragmentación por infraestructuras de transporte, elementos lineales que ocupan una reducida superficie aunque presentan una elevada intensidad de uso, 
aporta efectos particulares sobre el medio ambiente, tanto en fase constructiva como en fase de explotación. Dichos efectos operan en diferentes escalas y pueden actuar de forma sinérgica. Normalmente, las vías de alta capacidad ofrecen mayor impacto ya que toleran una mayor cantidad de vehículos. Sin embargo, el resto de vías, si bien no soportan altas cantidades de tráfico, se extienden por una mayor proporción de territorio, implicando igualmente un efecto negativo significativo sobre los ecosistemas.

El presente estudio se centra en la fragmentación causada por infraestructuras lineales de transporte por carretera. La implantación de las infraestructuras induce cambios en el territorio, tanto estructurales (urbanización) como funcionales (incremento del volumen del tráfico) (Ministerio de Medio Ambiente y Medio Rural y Marino, 2010), afectando además a la permeabilidad del paisaje, hecho que conlleva la creación de un efecto barrera (Forman et al., 2003; Ministerio de Medio Ambiente, 2006). Según afirma la Agencia Europea de Medio Ambiente (2004) la mitad de las áreas protegidas del continente europeo se ven ya sometidas a presiones ambientales por infraestructuras de transporte. Ante esta situación, se pone de manifiesto la necesidad de fomentar una infraestructura verde que permita el desplazamiento de las especies entre los diferentes espacios naturales protegidos, a través de la adopción de un enfoque integrado de la gestión del suelo y una cuidadosa planificación estratégica del territorio (Unión Europea, 2010). Del mismo modo, la evaluación de la situación actual de fragmentación de hábitats y la posterior propuesta de alternativas y medidas de gestión resulta de gran importancia para la conservación de la biodiversidad en el ámbito local y regional.

Este estudio pretende identificar dónde se produce la fragmentación de hábitats causada por infraestructuras lineales de transporte (descartando el transporte ferroviario y las sendas y pistas forestales) y evaluar su intensidad dentro de los límites insulares de Mallorca. La elaboración de este estudio pretende generar conocimiento sobre el estado de conservación de los hábitats mallorquines que pueda servir de herramienta de gestión para las administraciones, constituyendo un mecanismo eficiente para mitigar los efectos de las transformaciones (ecológicas) en la conservación de los valores naturales del territorio (Ministerio de Medio Ambiente y Medio Rural y Marino, 2010).

\section{2. Área de estudio}

La isla de Mallorca $\left(3.640 \mathrm{~km}^{2}\right)$, situada en el mar Mediterráneo occidental y ubicada entre las coordenadas $39^{\circ} 16^{\prime}$ - 39 $38^{\circ}$ ' $\mathrm{N}$ y $2^{\circ} 21^{\prime}$ - $3^{\circ} 29^{\prime} \mathrm{E}$, es la mayor del archipiélago Balear (73\% de la extensión total). Su configuración fisiográfica responde básicamente a tres unidades definidas (Moragues, 1993; Robledo, 2005; Giménez et al., 2014): (1) la Sierra de Tramuntana, que corresponde al horst noroccidental de Mallorca, donde se encuentran las mayores elevaciones orográficas (1445 m., Puig Major). (2) Las sierras de Levante, que corresponden al horst oriental de Mallorca, y están caracterizadas por altitudes menores. Ambos sistemas montañosos mantienen una disposición NESW que limitan un llano central o graben denominado "es Pla" (3). Esta configuración fisiográfica determina la organización territorial de la isla (infraestructuras, asentamientos urbanos, etc.) y los aspectos bióticos de flora y fauna. Geológicamente, en zonas de relieve predominan las rocas calizas y dolomíticas del Jurásico inferior (lías) y Triásico, 
mientras que en la llanura aluvial del Pla predominan arcillas del Mioceno y depósitos aluviales del Cuaternario. El sistema hidrográfico de Mallorca presenta la ausencia de cursos fluviales naturales permanentes, aunque en episodios lluviosos el agua es canalizada a través de torrentes que recorren prácticamente toda la isla. Asimismo, las aguas subterráneas suponen el 95\% de los recursos hídricos de las Baleares (Giménez et al., 2014). Climatológicamente, la existencia de un marcado período de déficit hídrico estival, la irregularidad y el régimen torrencial de las precipitaciones, y unas temperaturas suaves en invierno y muy cálidas en verano, caracterizan el clima mediterráneo propio de la isla de Mallorca, en la que los episodios de sequía meteorológica son habituales (Lorenzo-Lacruz y Morán-Tejada, 2016).

El presente estudio analiza los hábitats naturales afectados por la fragmentación causada por las carreteras que se encuentren dentro de áreas focales, entendiendo a las mismas como zonas que dispongan de una protección ambiental. Mallorca dispone de diversas figuras de protección ambiental organizadas, básicamente, en cuatro escalas competenciales: internacionales, europeas, estatales y autonómicas. Este estudio de fragmentación de hábitats, centra el análisis en la escala normativa europea, la cual aporta Red Natura 2000 compuesta por dos figuras de protección ambiental: Lugares de Interés Comunitario (LIC) figura de protección destinada a la conservación de la biodiversidad y Zonas de Especial Protección para las Aves (ZEPA) figura de protección específica para las aves y los hábitats donde nidifican. En Mallorca existen un total de 42 LICs y 29 ZEPAs.

A nivel biótico, en Baleares la flora autóctona asciende a 1729 taxones agrupándose en sólo 8 familias. Los terófitos son la forma vital más numerosa y la tasa de endemicidad es del 10\% de la flora autóctona (173 taxones). En Mallorca existen un total de 1445 taxones de flora y 1348 especies (Rita y Payeras, 2006). En cuanto a endemismos, Mallorca cuenta con 125 especies endémicas que se concentran en hábitats poco accesibles donde la presencia antrópica es escasa, como por ejemplo acantilados litorales y cimas montañosas. Las series de vegetación que coexisten en Mallorca se organizan de acuerdo con las variaciones en la distribución de los factores ecológicos como temperatura, humedad, salinidad, topografía, etc. (Llorens et al., 2007). La vegetación del litoral de Mallorca está caracterizada por sus adaptaciones a factores ecológicos como el viento, la salinidad o incluso la falta de suelo desarrollado. En ambientes halófilos como las albuferas y salobrales, la vegetación presenta una notable tolerancia a la salinidad dotando a sus especies de una presión osmótica alta que les permite contrarrestar la presencia de sal en sus tejidos. Estos hábitats litorales en ocasiones se combinan con zonas de chaparrales, pinares y sabinares en aquellas zonas donde la presión urbanística es menor. A medida que aumenta la distancia respecto de la costa los factores ecológicos litorales disminuyen y aparecen zonas dedicadas al cultivo, chaparrales y en ocasiones proliferan acebuchales fuertemente condicionadas por presiones urbanísticas. En zonas donde aumentan la altitud y la pendiente y, consecuentemente, disminuye la presión urbanística, irrumpen los encinares y pinares de Pinus halepensis.

A nivel faunístico, aunque la fragmentación de hábitats influye en todas las especies, el análisis se ha centrado en los vertebrados. Según la aportación de Viada (2006), los 
anfibios son sensibles a los cambios ambientales debido a su dificultad de dispersión y recolonización, sus restricciones fisiológicas, su baja movilidad y su doble ciclo de vida. Las condiciones ambientales de las islas no favorecen la supervivencia de los anfibios. Así pues, de las tres especies de anfibios presentes en Mallorca, tan sólo una es autóctona y endémica: el ferreret (Alytes muletensis), siendo las demás especies introducidas como consecuencia de la actividad humana. Probablemente, el factor de amenaza más grave es la pérdida, fragmentación y degradación del hábitat, difícil de controlar en islas turísticas como Baleares (Viada, 2006), así como la introducción de especies. La fauna silvestre de mamíferos de Baleares está formada por 32 especies, 20 de las cuales son murciélagos (Serra et al., 2011). El resto de especies han sido introducidas por el hombre, dando lugar incluso a procesos de subespeciación. En cuanto a las aves, son el grupo de vertebrados con más especies de las Baleares, con un total de 355. Existen dos especies endémicas: la pardela balear (Puffinus mauretanicus) y la curruca balear (Sylvia balearica). Las principales causas de extinción de la avifauna consideradas son la presencia de especies alóctonas invasivas y la pérdida y degradación de hábitats.

En cuanto a la infraestructura de carreteras de la isla de Mallorca (Fig. 1), ésta dispone de 5959 kilómetros (2012) de longitud. La red sigue una estructura radial, de manera que la mayoría de vías de la isla -particularmente las vías de alta capacidadconvergen en el área metropolitana de Palma, siendo ésta el área central funcional, administrativa y poblacional de Mallorca (Rodríguez y Gutiérrez, 2012). Las vías de

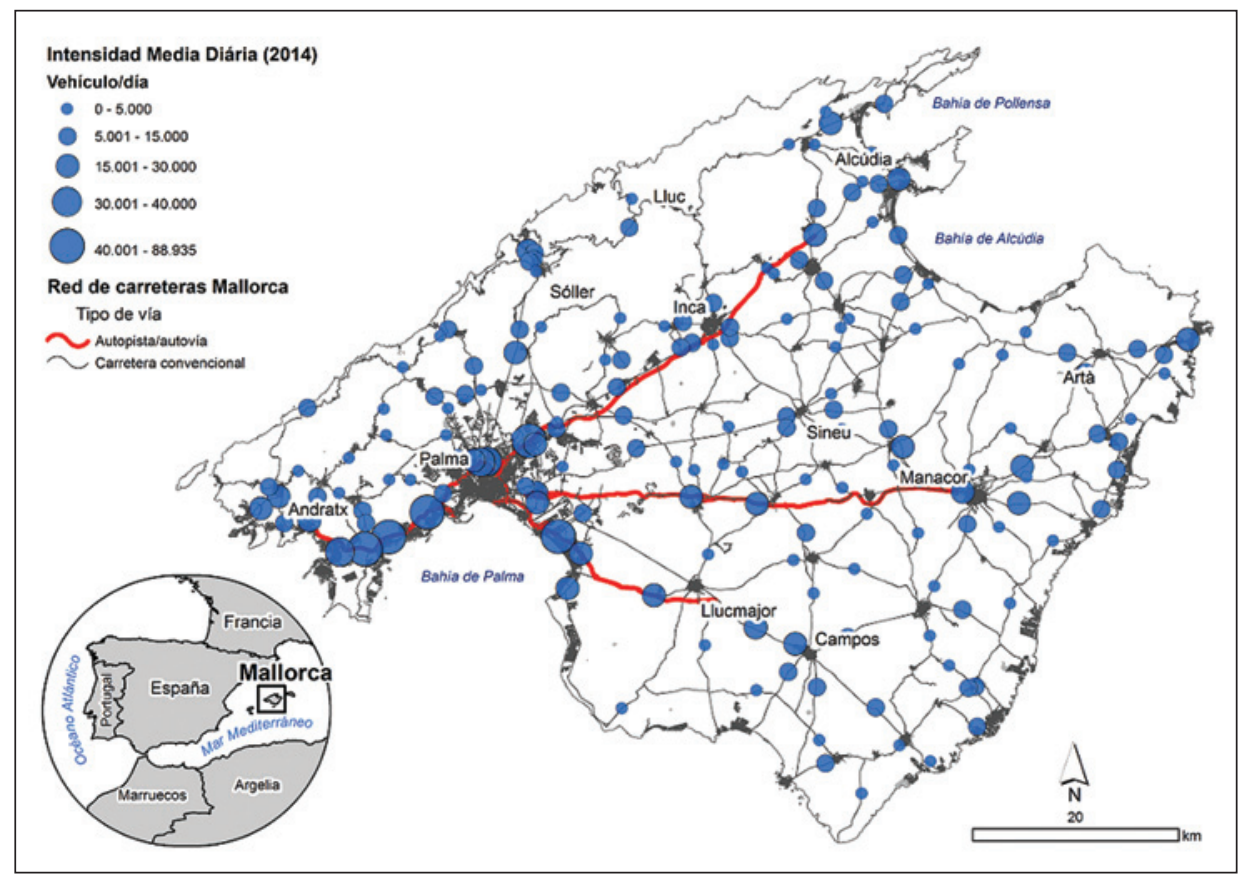

Figura 1. Red de carreteras de Mallorca y tráfico rodado (Intensidad Media Diaria registrada en el año 2014). 
alta capacidad que unen Palma con Peguera (Ma-1), sa Pobla a través del eje del Raiguer (Ma-13), Llucmajor (Ma-19) y Manacor (Ma-15), jerarquizan la red de carreteras de Mallorca. Ésta presenta mayor densidad en las inmediaciones de la ciudad de Palma, debido a su carácter neurálgico, y en el centro de la isla coincidiendo con una fisiografía suave y relieve poco pronunciado. Por el contrario, las zonas con relieve abrupto como el centro y norte de la sierra de Tramuntana, la densidad de la red es menor, al igual que en la zona de la marisma de Llucmajor.

Los aforamientos de IMD (Intensidad Media Diaria) muestran una clara confluencia de las mayores densidades alrededor de la capital, canalizados a través de vías de alta capacidad que se extienden jerarquizando las carreteras de la isla, con valores que oscilan entre 40.000 y 90.000 vehículos/día. Las demás carreteras convencionales presentan intensidades que no suelen rebasar los 30.000 vehículos/día. En zonas abruptas donde transcurren carreteras sinuosas y/o estrechas las densidades son menores, como por ejemplo en la Sierra de Tramuntana o en carreteras secundarias del interior de Mallorca.

\section{Materiales y métodos}

La fragmentación de hábitats comprende procesos complejos que tienen afectación sobre aspectos diversos del paisaje. Para la realización del presente trabajo se ha seguido la metodología propuesta por el Ministerio de Medio Ambiente y Medio Rural y Marino (2010), consistente en la aplicación de una serie de indicadores basados en parámetros fisiográficos y morfológicos, relacionados con la fragmentación de hábitats causada por infraestructuras lineales de carreteras, a través de su integración en un Sistema de Información Geográfica (SIG).

Tabla 1. Información geográfica utilizada en el estudio: procedencia y descripción.

\begin{tabular}{|c|c|c|c|}
\hline $\begin{array}{l}\text { Nombre } \\
\text { (Fecha de } \\
\text { creación) }\end{array}$ & Fuente & Características & Escala \\
\hline LIC (2015) & $\begin{array}{l}\text { Banco de Datos de la } \\
\text { Naturaleza }\end{array}$ & $\begin{array}{l}\text { Capa de información vectorial poligonal } \\
\text { que muestra la localización de las zonas } \\
\text { declaradas LIC de Baleares. }\end{array}$ & $1: 50.000$ \\
\hline ZEPA (2015) & $\begin{array}{l}\text { Banco de Datos de la } \\
\text { Naturaleza }\end{array}$ & $\begin{array}{l}\text { Capa de información vectorial poligonal } \\
\text { que muestra la localización las zonas de- } \\
\text { claradas ZEPA de Baleares. }\end{array}$ & $1: 50.000$ \\
\hline $\begin{array}{l}\text { Red de } \\
\text { Carreteras } \\
(\mathbf{2 0 1 2})\end{array}$ & Consell de Mallorca & $\begin{array}{l}\text { Capa de información vectorial lineal que } \\
\text { representa las carreteras convencionales, } \\
\text { autopistas y autovías de Baleares. }\end{array}$ & $1: 5000$ \\
\hline $\begin{array}{l}\text { Intensidad } \\
\text { Media Diaria } \\
(\mathbf{2 0 1 4})\end{array}$ & Consell de Mallorca & $\begin{array}{l}\text { Capa de información vectorial de multi- } \\
\text { puntos que contiene el número medio de } \\
\text { vehículos que pasan por las } 170 \text { estacio- } \\
\text { nes de aforo localizadas en las carreteras } \\
\text { de Mallorca. }\end{array}$ & $1: 5000$ \\
\hline
\end{tabular}


Partiendo de la cartografía básica descrita anteriormente (Tabla 1), los distintos análisis realizados han permitido la modelización y generación de nuevas capas de información espacial y la obtención de numerosos resultados orientados a la consecución de los objetivos planteados. Posteriormente se realizó una selección de los indicadores más representativos sobre el estado de fragmentación actual de la isla de Mallorca, propuestos por el Ministerio de Medio Ambiente y Medio Rural y Marino en el informe técnico "Indicadores de fragmentación de hábitats causada por infraestructuras lineales de transporte" (2010). Los indicadores presentados en el análisis están incluidos en tres grupos jerarquizados según su complejidad creciente:

\subsection{Medidas sobre las infraestructuras}

Aportan información indirecta sobre la afectación ecológica de las infraestructuras de transporte en la fragmentación de los hábitats.

\subsubsection{Superficie ocupada por infraestructuras de transporte}

Este indicador resulta del cálculo de la superficie (en hectáreas) ocupada por infraestructuras, diferenciando autopistas/autovías de las carreteras convencionales, considerando una anchura media de 35 y 14 metros respectivamente.

\subsubsection{Intensidad media de uso}

Se determina la cantidad de vehículos que transitan diariamente por las vías, quedando registrados en las estaciones de aforamiento de vehículos que se encuentren dentro o a 300 metros de un área focal LIC o ZEPA.

\subsubsection{Intersección con las infraestructuras}

Este indicador muestra la longitud (en metros) de infraestructura de carreteras que penetran dentro de áreas focales o de especial interés.

\subsection{Medidas sobre las propiedades topológicas de los hábitats}

Aportan información sobre las características morfométricas de los hábitats, su cohesión y la intensidad del efecto borde que les afecta.

\subsubsection{Tamaño de las teselas}

Este indicador topológico surge directamente del concepto más puro de fragmentación (Ministerio de Medio Ambiente y Medio Rural y Marino, 2010) y consiste en la medición de las superficies (en hectáreas) de los LIC y ZEPA considerados en el presente análisis como área focales.

\subsubsection{Forma de las teselas}

Algunos indicadores de fragmentación miden aspectos de forma como la relación área/perímetro a través de medidas diversas (Ministerio de Medio Ambiente y Medio Rural y Marino, 2010). En este trabajo se ha utilizado el perímetro normalizado $(P n)$ 
que, a diferencia del tradicional ratio área/perímetro, resume de forma más ajustada las características morfológicas de las teselas y es resultado de:

$$
P n=\frac{P}{P^{\prime}}
$$

donde $P$ corresponde al perímetro de la tesela y $P^{\prime}$ al perímetro circular del mismo área que la tesela de estudio, dado que el círculo es la forma geométrica con una menor relación área/perímetro. Los valores inferiores a 1 representan aquellas áreas focales donde el perímetro real $(P)$ es inferior al perímetro circular para la misma área $\left(P^{\prime}\right)$, mientras que un valor superior a 1 representa lo contrario. Por lo tanto, se considera que los valores alejados a 1 tienden a formas más sinuosas y menos compactas o cohesionadas.

\subsubsection{Superficie con efecto borde}

El efecto borde aumenta debido a la implantación de infraestructuras de transporte que atraviesan áreas focales, reduciendo así la relación área/perímetro y aumentando la incidencia de las perturbaciones externas. Precisamente, este indicador mide la superficie de borde teniendo en cuenta la irrupción de infraestructuras de transporte. Aunque el efecto borde varía dependiendo de las condiciones de cada hábitat y de las características de cada carretera, según la bibliografía consultada (Rosell et al., 2003), se recomienda establecer un efecto borde entre 200 y 500 metros para el borde del área focal y un efecto borde de hasta 400 metros para las carreteras. De esta manera, y para unificar criterios, se estableció un borde alrededor del área focal de 300 metros y otro borde para cada lado de la carretera de 200 metros.

\section{Resultados}

La fragmentación de hábitats es un proceso complejo que engloba un gran número de patrones y efectos muy diversos sobre el medio ambiente, difícilmente medibles (Ministerio de Medio Ambiente y Medio Rural y Marino, 2010). No obstante, los indicadores permiten realizar una aproximación relativamente certera a la realidad. A continuación se exponen los resultados obtenidos a partir de la aplicación de los indicadores propuestos.

\subsection{Medidas sobre las infraestructuras}

\subsubsection{Superficie ocupada por las infraestructuras}

Mallorca cuenta con 7906,96 hectáreas ocupadas por infraestructuras de transporte por carretera $(2,17 \%$ de la superficie de la isla), de las cuales 876,84 corresponden a vías de alta capacidad, mientras que 7030,12 corresponden a carreteras convencionales. El $31 \%$ de la superficie total de Mallorca presenta algún tipo de protección medioambiental, ya sea LIC o ZEPA. De esta superficie, 176,52 ha. LIC se destinan a vías convencionales, mientras que los espacios ZEPA son atravesados por 130,57 hectáreas de carreteras convencionales. No existe ningún espacio declarado como LIC o ZEPA atravesado por vías de alta capacidad (Tabla 2). 
Tabla 2. Superficie de hábitat ocupada por las infraestructuras de transporte en Mallorca.

\begin{tabular}{lrrrrrrr}
\hline \multicolumn{7}{c}{ Superficie ocupada por las infraestructuras } & \\
& \multicolumn{1}{c}{ Sup. } & \% Sup. & LIC & \% LIC & ZEPA & \% ZEPA \\
\hline Autopista/Autovía & 876,84 ha. & $0,24 \%$ & 0 & $0 \%$ & 0 & $0 \%$ \\
C. convencional & 7030,12 ha. & $1,93 \%$ & 176,52 ha. & $0,29 \%$ & 130,57 ha. & $0,24 \%$ \\
Mallorca & 363.570 ha. & $100 \%$ & 60.351 ha. & $100 \%$ & 53.579 ha. & $100 \%$ \\
\hline
\end{tabular}

\subsubsection{Intensidad media de uso de las infraestructuras}

En cuanto a la intensidad de tráfico registrada en las carreteras que transcurren por espacios declarados LIC o ZEPA, o a menos de 300 metros, la media de presión es de 5500 vehículos/día. Se pueden observar registros superiores a los 10.000 vehículos/día en 4 de los 18 puntos (Fig. 2A), localizados en torno a la costa nororiental de Mallorca (Ma-12 y Ma-2220) y en la península de Artà (Ma-15). A su vez se observan intensidades de tráfico de magnitud media y alta en los espacios protegidos de Tramuntana. Por el contrario, en espacios situados al sur de Mallorca la intensidad media de uso de las infraestructuras disminuye por debajo de los 1000 vehículos/día. La Albufera de Alcudia es el espacio natural protegido que recibe una mayor intensidad de uso, con 14.825 vehículos/día, mientras que el espacio natural protegido de Ses Salines presenta una presión de 70 vehículos/día.

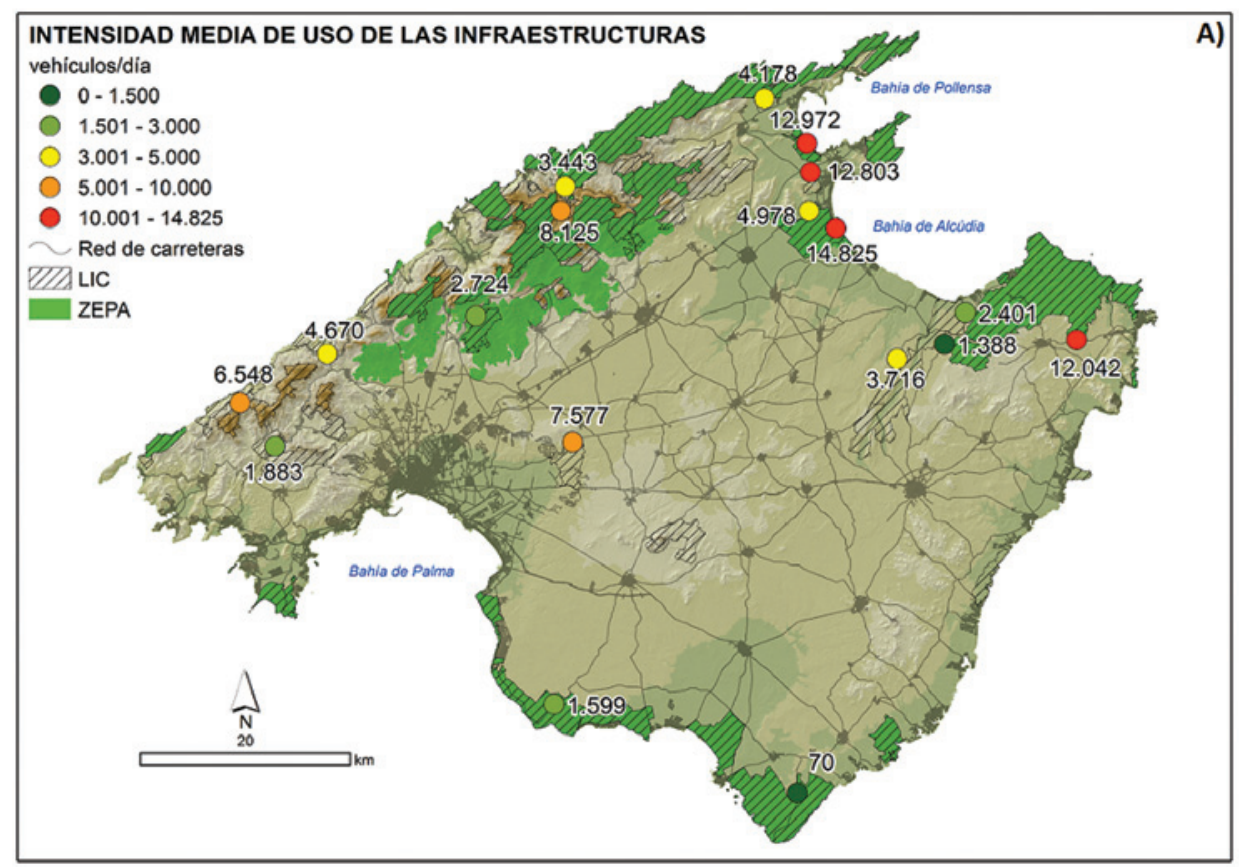

Figura 2. A) Intensidad media de uso de las infraestructuras de Mallorca según datos del departamento de carreteras del Consell de Mallorca (2014). 


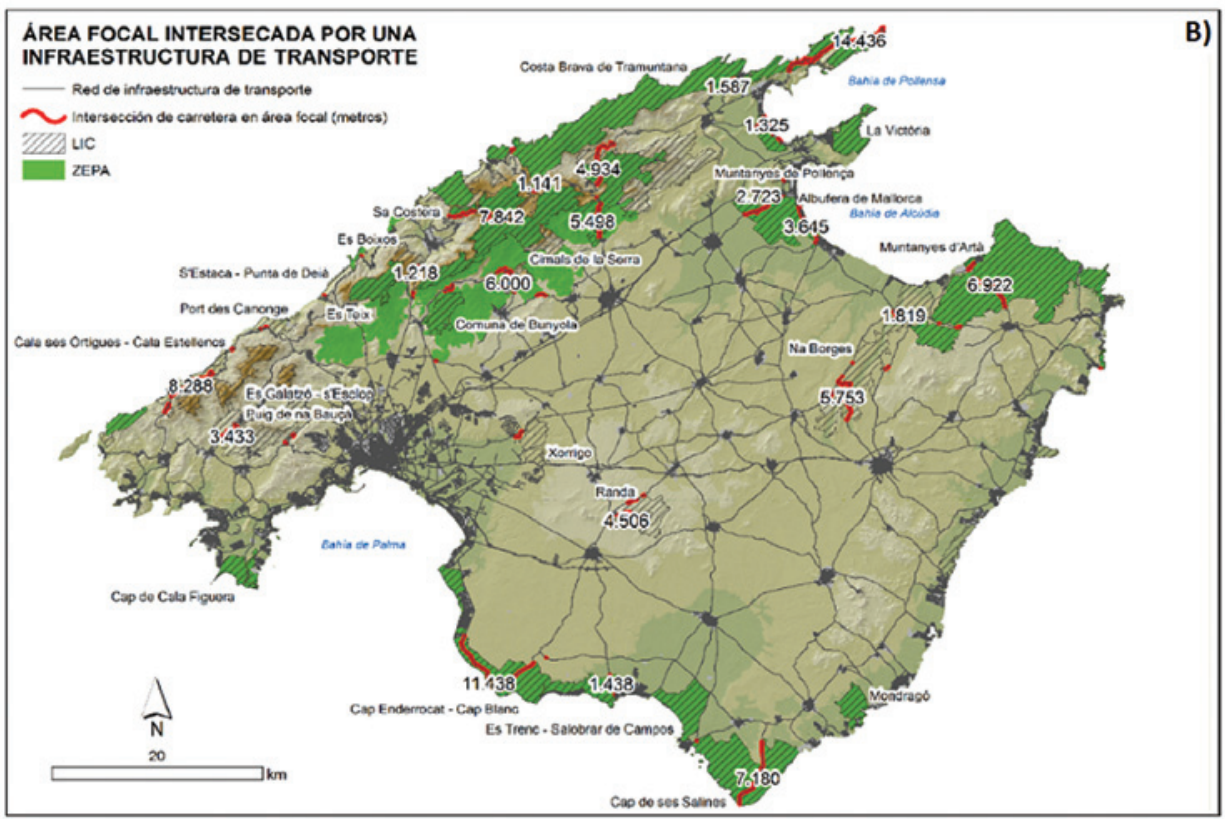

Figura 2. B) Área focal intersectada por una infraestructura de transporte.

\subsubsection{Intersección con las infraestructuras}

La figura 2B muestra la longitud de los tramos de carretera que intersectan con algún hábitat protegido. Un total de 112.369 metros de carreteras intersectan un área focal LIC (102.658 m.) o ZEPA (75.882 m.), teniendo en cuenta que 66.171 metros transcurren en ambos espacios. Esto supone que un 5,3\% del total de la red de carreteras, intersectan con un área focal. Al noroeste de Mallorca, coincidiendo con la sierra de Tramuntana, se observa una mayor intersección de áreas focales, puesto que se trata de un espacio altamente protegido por diversas figuras de protección. De media, los espacios protegidos se encuentran intersectados por 1887 metros de infraestructura viaria. En cuanto a los valores extremos, el área focal intersectada con más metros de infraestructura es Formentor, con un total de 14.436 metros de infraestructura de carretera, seguido de Cap Enderrocat-Cap Blanc, con 11.438 metros de carretera. En la parte central de la sierra de Tramuntana se observa una desigual intersección longitudinal de las infraestructuras, con valores comprendidos entre los 1000 y 8000 metros.

\subsection{Medidas sobre las propiedades topológicas de los hábitats}

\subsubsection{Tamaño de las teselas}

La figura 3A muestra el tamaño de las áreas bajo la figura de protección LIC. Siguiendo el mismo patrón general que el indicador anterior, la sierra de Tramuntana presenta cierta complejidad. Particularmente, se observa una mayor concentración de 

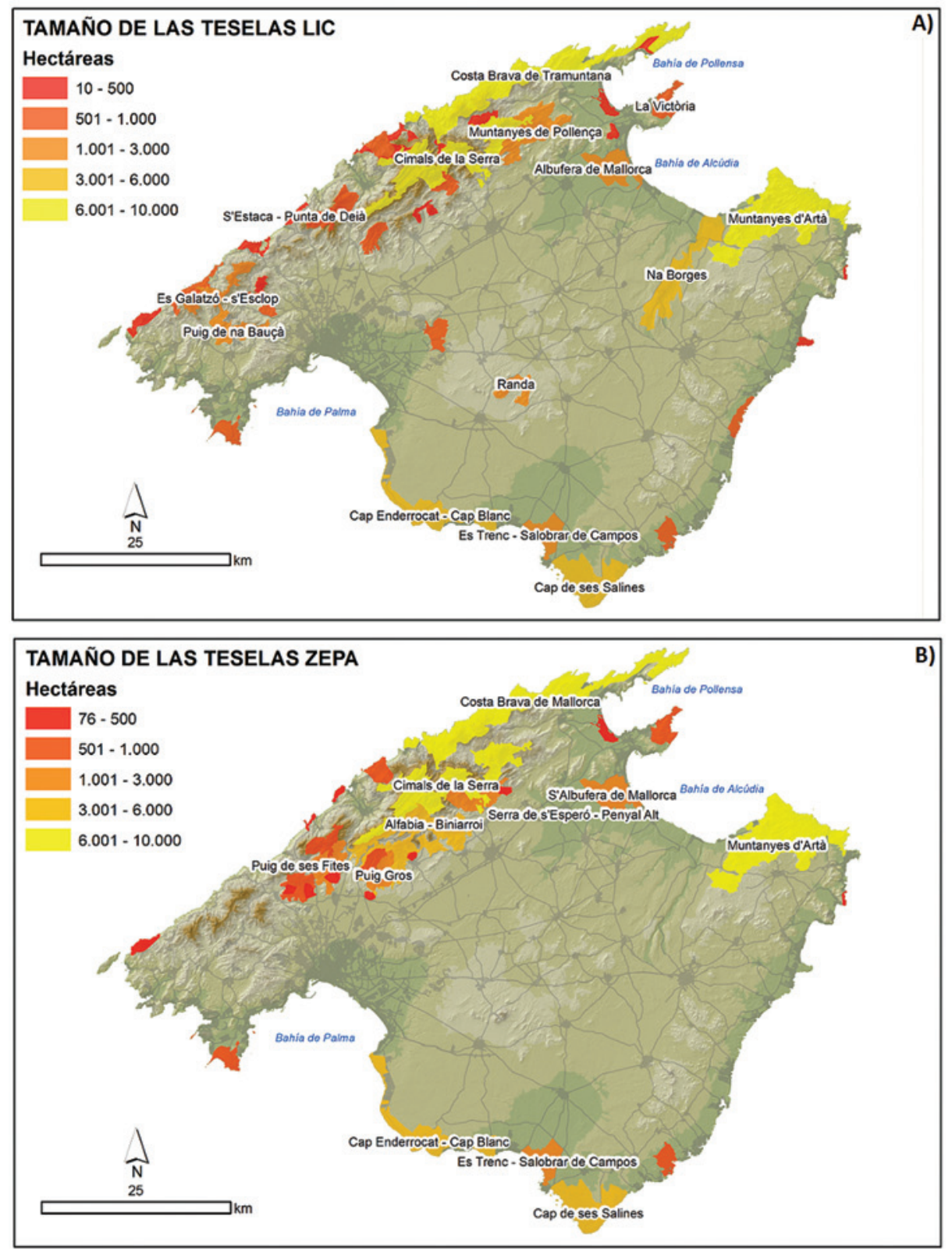

Figura 3. A) Tamaño de las teselas LIC.

B) Tamaño de las teselas ZEPA, ambas expresadas en hectáreas.

LICs de pequeñas dimensiones, algunos de ellos limítrofes entre sí, en la zona más occidental de la isla. Destacan los LICs de Monnàber y Es Binis con una superficie inferior a 100 hectáreas. En cuanto a los ZEPAs (Fig. 3B), solamente destaca el de Cap 
Vermell, situado en la vertiente litoral oriental, que cuenta con una superficie de 75 ha., siendo éste el único espacio ZEPA con una superficie inferior a 100 hectáreas. Las teselas con mayores superficies se localizan en la Costa brava de Mallorca (8310 ha.) y en las Muntanyes d'Artà (9085 ha.), coincidiendo tanto LICs como ZEPAs. En la sierra de Tramuntana, ambas tipologías de espacios naturales protegidos presentan superficies extensas al noreste, viéndose gradualmente disminuidas sus dimensiones a medida que se localizan más al suroeste.

\subsubsection{Forma de las teselas}

La figura 4 representa una medida del grado de sinuosidad de los hábitats mallorquines catalogados como LIC y ZEPA (A y B respectivamente). El perímetro normalizado medio de los espacios naturales catalogados como LIC es de 1,13, presentando una amplitud entre el valor más bajo y el más alto de 0,87 . Las teselas que presentan un perímetro normalizado próximo a 1 y por lo tanto tienen una forma poco sinuosa, más cohesionada, son los LICs Serra d'en Salat $(0,97)$ y Sa Costera $(1,02)$. Por el contrario, los LICs de Cimals de la Serra y Muntanyes d'Artà presentan los valores de perímetro normalizado más alejados a $1 \mathrm{y}$, por consiguiente, tienden a formas más sinuosas. El perímetro normalizado con un valor más bajo corresponde al LIC s'EstacaPunta de Deià $(0,77)$.

En cuanto a los ZEPAs el perímetro normalizado medio se encuentra en 1,20 y la amplitud de sus valores es de 1,94, por tanto presentan unas formas más variadas y

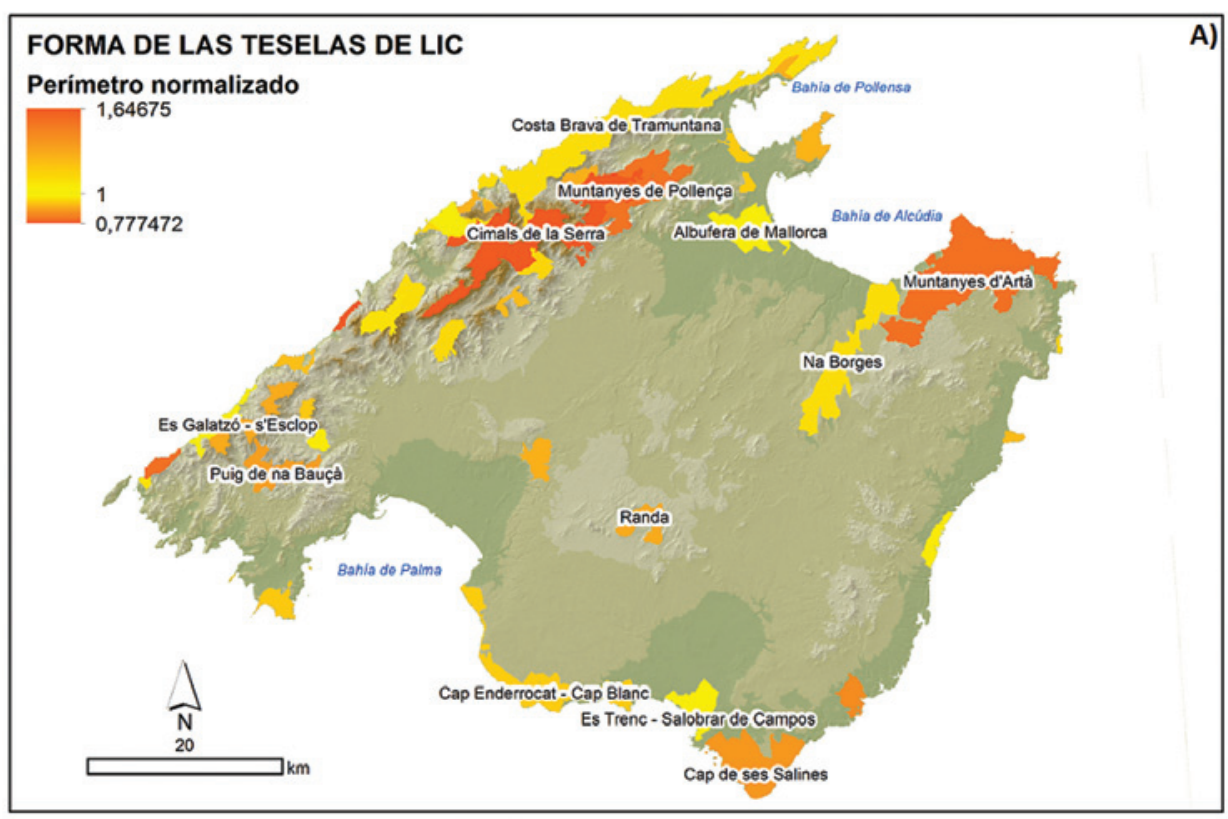

Figura 4. A) Forma de las teselas de LIC a partir del perímetro normalizado. 


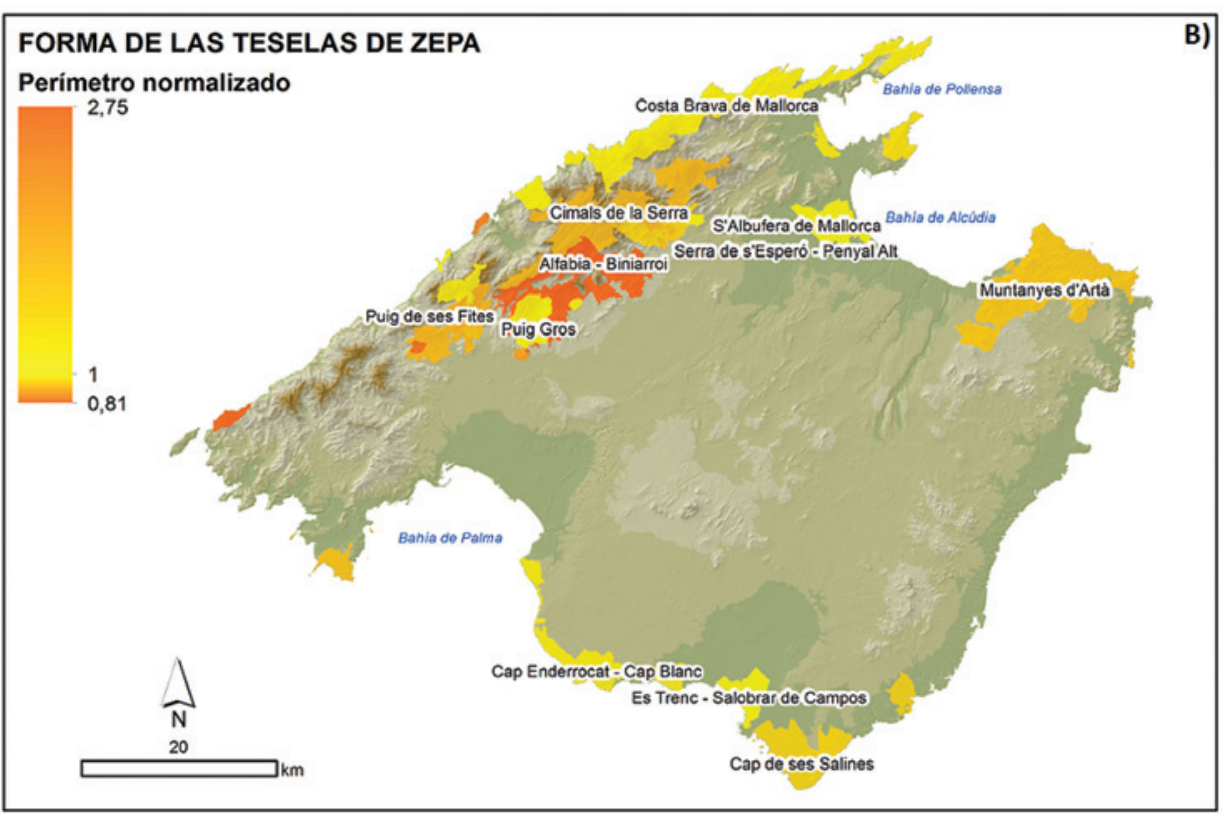

Figura 4. B) Forma de las teselas ZEPA a partir del perímetro normalizado.

menos cohesionadas que los LICs. Las áreas focales que presentan valores próximos a 1 son Cap Vermell $(0,95)$ y Sa costera $(1,02)$. Por el contrario, destaca la sinuosidad de la ZEPA Alfabia-Biniarroi la cual presenta un perímetro normalizado de 2,75 que denota su significativa sinuosidad, seguido del Puig de ses Fites $(1,77)$. El valor más bajo del perímetro normalizado es el correspondiente a La Trapa $(0,81)$.

\subsubsection{Superficie con efecto borde}

La figura 5 muestra una representación cartográfica de las zonas LIC y ZEPA (A y B respectivamente) afectadas por los procesos asociados a los bordes de hábitats. Los LICs presentan una superficie total de 60.351 hectáreas, con una superficie de borde sin infraestructuras de transporte de 25.243,86 hectáreas. Esto supone que el $42 \%$ de la superficie de los LICs son espacios con efecto borde. Con la implantación de infraestructuras de carretera, la superficie total del borde de hábitat aumenta un 3,5\% (2130 hectáreas). Tal y como se puede observar en el mapa de la figura 5A, los LICs más afectados por el efecto borde, en valores relativos, son Es Binis y Cap Vermell (ambos 100\%), mientras que el LIC menos afectado es Sa Costera, con un 24,9\% de superficie con efecto borde.

Los ZEPAs presentan una superficie total de 53.570 hectáreas y una superficie de borde $\sin$ infraestructuras de transporte de 20.650 , lo que supone que el $38 \%$ de la superficie de ZEPA son espacios borde. Con la implantación de infraestructuras de carretera, el borde de hábitat aumenta un 2,4\% (1298 hectáreas). En este sentido, el espacio ZEPA más afectados por el efecto borde en valores relativos es Cap Vermell, con una afectación de 
borde del 100\%, mientras que el ZEPA Comuna de Bunyola es el menos afectado, con un $13,8 \%$ de efecto borde (Fig. 5B). Tanto en espacios LIC como en ZEPA, alrededor del $60 \%$ (de media) de la superficie de los espacios presentan efecto borde.

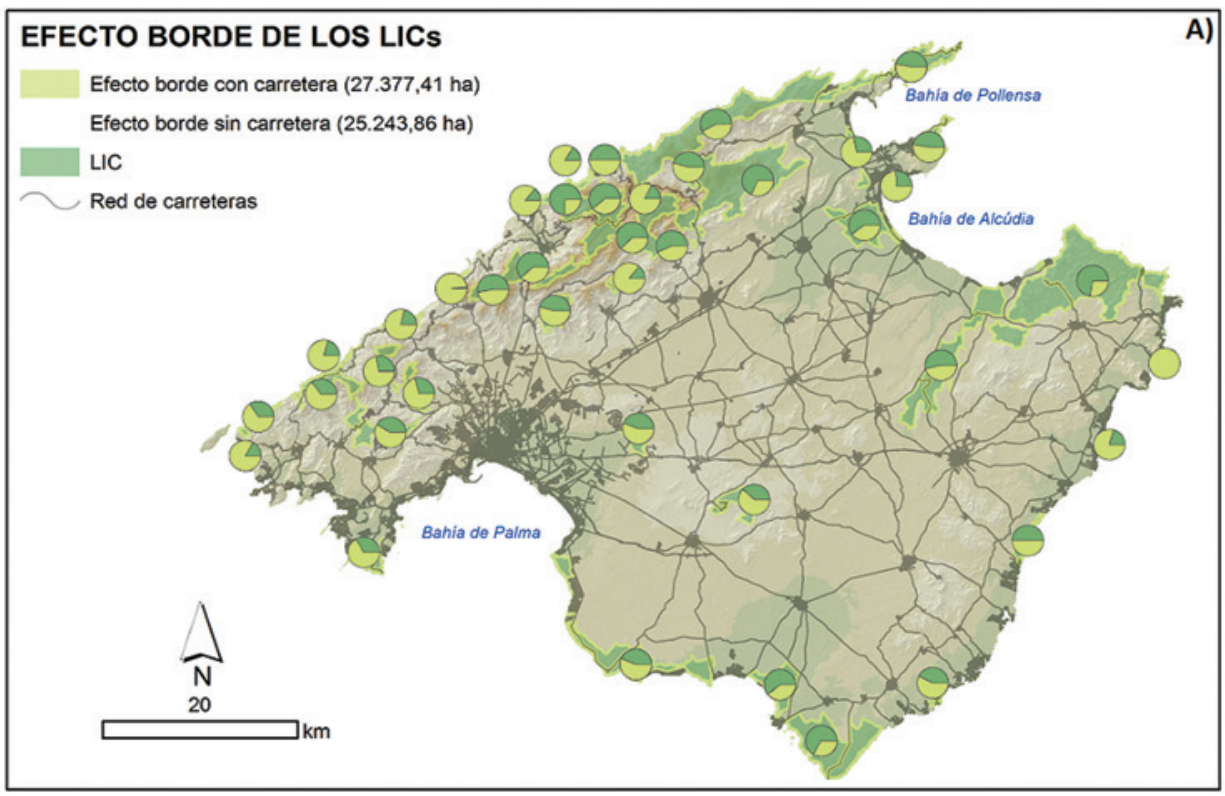

\section{EFECTO BORDE DE LOS ZEPAS}

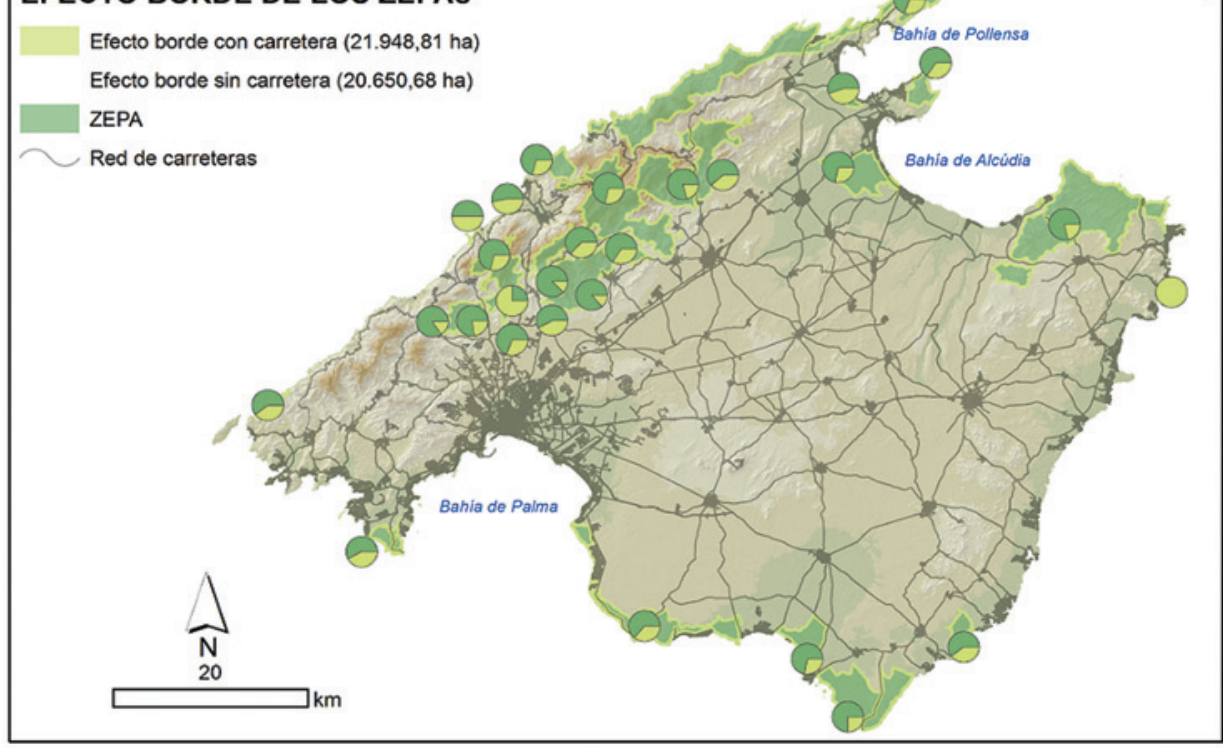

Figura 5. A) Proporción de hábitat de borde respecto al hábitat interior de las teselas LIC. B) Proporción de hábitat de borde respecto al hábitat interior de las teselas ZEPA. 


\section{Discusión}

Durante las últimas décadas, la dinámica de los paisajes europeos se ha traducido, en multitud de casos, en un aumento de la fragmentación y reducción de hábitats (Gurrutxaga y Lozano, 2006). La isla de Mallorca se encuentra entre esos espacios afectados, ya que como consecuencia de la presencia humana intensiva y ancestral, prácticamente todo el territorio presenta algún tipo de alteración, dando lugar a un complejo mosaico paisajístico (Llorens et al., 2007; Morera et al., 2007). La fragmentación se manifiesta como un patrón dentro del espacio geográfico (Gurrutxaga y Lozano, 2006), y por este motivo, los procesos de fragmentación son cada vez más estudiados en diferentes disciplinas. Los conceptos mosaico y elementos del paisaje representan la base de la interpretación del paisaje, pudiendo aplicarse o inferir a cualquier escala (Vila et al., 2006). En este sentido, la fragmentación, como proceso eminentemente espacial, tiene una escala específica de operatividad (García, 2011). En el presente análisis sobre el estado de fragmentación de los hábitats de Mallorca, la escala de trabajo elegida viene determinada por los límites físicos impuestos por la propia isla, a diferencia de otros estudios donde los límites del análisis se determinan por otro tipo de factores fisiográficos o ecológicos.

En este estudio se aborda el problema de la fragmentación de hábitats desde la perspectiva de los impactos ocasionados por las infraestructuras viarias de transporte. De Montis et al. (2017) desarrollaron varios índices, distintos a los aquí utilizados, para evaluar el grado de fragmentación del paisaje causado por el desarrollo urbano y de infraestructuras en dos regiones (Andalucía y Cerdeña) de la Europa Mediterránea. Los resultados coinciden con los del presente trabajo, al detectar las mayores afecciones por fragmentación en áreas costeras. Otros trabajos en el ámbito internacional se han servido de indicadores similares a los aquí utilizados para detectar y cuantificar la fragmentación de hábitats en el valle de Adige en Italia (Geneletti, 2004), isla de Wight en Inglaterra (Quine y Watts, 2009) o Estados Unidos (Riitters et al., 2004). En el caso de Mallorca, las infraestructuras viarias de transporte ocupan una superficie de poco menos del 2,2\%. Incluso en espacios naturales protegidos (LIC y ZEPA), el porcentaje de superficie destinado a infraestructuras es inferior al 1\%. A simple vista estos valores denotarían una baja afectación de las vías en procesos de fragmentación. No obstante, otros indicadores como la intensidad de uso o la intersección de vías penetrantes en estos espacios naturales esclarecen unos impactos asociados mucho más notables. Por una parte, la intensidad media de uso de las vías está fuertemente condicionada por la estacionalidad turística. Los espacios naturales cercanos a núcleos turísticos de costa, como Alcudia-Ca'n Picafort o Cala Ratjada, y a monumentos naturales o culturales, como el monasterio de Lluc, Sa Calobra o Cap de Formentor, presentan intensidades de uso que estarían asociadas al flujo extra de vehículos generado por el turismo durante los meses de temporada alta. Por otra parte, las características de la vía podrían condicionar estos flujos de vehículos. En general, las vías que cuentan con amplios carriles de circulación, poca sinuosidad (lo que favorece el flujo de vehículos) y transcurren por zonas poco desniveladas tienden a la acumulación de mayores flujos que las vías que cuentan con pocas prestaciones o son más sinuosas. 
La reducción del área neta y funcional del ecosistema, así como el aumento del aislamiento y la proliferación de zonas marginales, son factores de riesgo importantes de la fragmentación de hábitats (Sánchez y Calvo, 1999; Delgado et al., 2004). La superficie de los fragmentos muestra una clara correlación con la diversidad de especies que puede albergar. En este sentido, la teoría de la isla biogeográfica desarrollada por MacArthur y Wilson (1963 y 1967) determina una reducción progresiva de la diversidad biológica, así como de la dimensión de las poblaciones de las diferentes especies presentes, fruto de la reducción de la extensión de los fragmentos (Vila et al., 2006). Así pues, según los resultados obtenidos en el análisis realizado, la zona oeste de Mallorca concentra los fragmentos de hábitat con menor superficie si se compara con la zona nordeste. Este patrón podría estar relacionado con el rápido crecimiento urbanístico (Mallarach y Marull, 2006) y podría estar reduciendo o limitando la diversidad potencial de especies en estas zonas.

La forma de los fragmentos es un indicador fundamental, considerado en algunas ocasiones más relevante que los indicadores de superficie (Vila et al., 2006). La forma está condicionada tanto por la actividad humana, lo que supone una mayor presencia de formas rectilíneas, como por las condiciones naturales (topografía, litología, etc.), éstas asociadas a formas curvilíneas e irregulares (Vila et al., 2006). Asimismo, la forma de las teselas está también relacionada con cambios en el efecto borde asociado frecuentemente a ésta (Ministerio de Medio Ambiente y Medio Rural y Marino, 2010). Según Sánchez y Calvo (1999), autores de un estudio sobre la distribución de aves en relación con la composición del paisaje en hábitats mediterráneos semiáridos de la región de Murcia, el efecto borde puede afectar negativamente a las zonas de nidificación de distintas especies de aves. Asimismo, la forma de las teselas influye en procesos que tienen lugar entre las mismas, como la migración de pequeños mamíferos terrestres, la colonización de algunas plantas o las estrategias alimentarias de algunas especies (Martín et al., 2006). Por tanto, las teselas que presentan formas más cohesionadas son menos sensibles a perturbaciones externas. En este caso, las formas de los espacios considerados LIC y ZEPA podrían estar determinadas por la complejidad del propio paisaje, puesto que las zonas centrales de la Sierra de Tramuntana se caracterizaran por perímetros normalizados sinuosos y poco cohesionados. Para el análisis morfológico se ha considerado pertinente utilizar el perímetro normalizado, ya que facilita la comprensión de la relación área/perímetro a nivel morfológico y funcional (Vila et al., 2006). Marín et al. (2008) realizaron un análisis sobre la fragmentación de los hábitats Red Natura 2000 afectados por el PEIT. No obstante, el análisis se circunscribía a la España peninsular, hecho que ha imposibilitado la comparación con los resultados de forma obtenidos en el presente análisis. De todos modos, ambas metodologías concluyen que cuanto mayor sea esta relación, mayor es la complejidad de las teselas y consecuentemente mayor es la fragmentación y sus impactos (Martín et al., 2006).

La superficie bajo efecto borde permite aproximar el estado del hábitat interior, que es fundamental para la presencia y mantenimiento de flora y fauna especialista, es decir, más exigente en sus requerimientos ecológicos (Forman y Godron, 1986 y Forman, 1995). Contrariamente, en hábitats de borde se favorece la presencia de especies generalistas o incluso invasoras, muchas de las cuales son depredadoras oportunistas que 
podrían dañar severamente el hábitat remanente (Bennet, 1991). Según un informe de la Comisión Europea, el 60\% de los hábitats de la UE presentan efecto borde (Artificial edge interface), incrementándose la proporción en la Europa mediterránea (Estreguil et $a l ., 2012)$. En Mallorca, la superficie de efecto borde de la Red Natura 2000 es del $43 \%$.

Es importante destacar algunas consideraciones que han condicionado el desarrollo conceptual y metodológico de este trabajo. En primer lugar, cabe reconocer que la información aportada por los indicadores utilizados es cuantitativa, hecho que dificulta la evaluación del estado de fragmentación de los hábitats, ya que se obvian situaciones de transición propias de la heterogeneidad existente en procesos naturales. En este mismo sentido, García (2011) asegura que existen demoras en la manifestación de los efectos de fragmentación que pueden provocar la valoración errónea de algunos indicadores.

Por otra parte, existe un notable déficit de información de comportamientos espaciales biológicos y de registros de mortalidad animal por atropello que puedan asegurar unos resultados inequívocos. Por esta razón, sería interesante la realización de campañas de muestreo para la recopilación y tratamiento de dichos datos, así como realizar el análisis a diferentes escalas temporales a fin de considerar las fluctuaciones anuales como, por ejemplo, las de la intensidad del tráfico. De esta manera se podrían considerar aspectos relacionados con la estacionalidad característica de las Baleares y su impacto en los procesos de fragmentación, para así poder implementar más y más eficientes medidas de gestión preventiva.

\section{Conclusiones}

La fragmentación de hábitats causada por infraestructuras viarias es una de las principales causas de la crisis actual de biodiversidad. En el caso de Mallorca, la proliferación de infraestructuras en espacios insulares limitados puede promover la atomización de los componentes del medio biótico. Los resultados obtenidos de la aplicación de los indicadores propuestos en el presente trabajo revelan un importante impacto de los procesos de fragmentación en espacios donde la intensidad media de uso y la proximidad de núcleos urbanos o turísticos son altas (especialmente al norte de la isla y en la Sierra de Tramuntana).

Las aportaciones realizadas en el presente estudio están encaminadas hacia el desarrollo de una planificación sostenible a nivel local y regional en las Islas Baleares, que considere los impactos de la movilidad sobre el medio ambiente y favorezca el mantenimiento y preservación de sus hábitats y espacios singulares. Igualmente, los resultados obtenidos tienen un gran valor potencial para la aplicación de políticas de conservación ambiental que minimicen los impactos de las actividades humanas en un paisaje caracterizado por la alta fragilidad propia de los sistemas insulares. Además, dicho análisis resulta una contribución valiosa y original, ya que esta problemática nunca antes había sido tratada en las Baleares desde esta perspectiva. El análisis del estado en que se encuentren los hábitats incluidos en Red Natura 2000, en un área de estudio sometida a una gran presión turística y urbanística, es fundamental para asegurar la conservación de la riqueza ecológica Balear. 


\section{Agradecimientos}

Los autores quieren agradecer la asistencia y asesoramiento prestados por el Dr. Guillem Xavier Pons Buades y por el Dr. Miquel Mir Gual en cuestiones de índole biológica, y al departamento de carreteras del Consell de Mallorca y al SSIGT de la Universidad de las Islas Baleares por la cesión de la información geográfica utilizada en este estudio. Gracias a los dos revisores anónimos por sus comentarios y sugerencias, todos ellos encaminados a mejorar la calidad final del trabajo.

\section{Referencias}

Bennet, A.F. 1991. Roads, roadsides and wildlife conservation: a review. In: D.A. Saunders, R.J. Hobbs (Eds.), Nature Conservation 2: The role of corridors. Surrey Beatty, Australia, pp. 99-118.

Brook, B.W., Sodhi, N.S., Ng, P.K.L. 2003. Catastrophic extinctions follow deforestation in Singapore. Nature 424, 420-423. http://doi.org/10.1038/nature01795.

Delgado, J.D., Arévalo, J.R., Fernández-Palacios, J.M. 2004 . Consecuencias de la fragmentación viaria: Efectos de borde de las carreteras en la laurisilva y el pinar de Tenerife. In: J.M. Fernández-Palacios, C. Morici (Eds.), Ecología insular/Island ecology. Asociación Española de Ecología Terrestre (AEET), Cabildo Insular de La Palma, pp. 181-225.

Del Barrio, G., Moral, R.G., Simón, J.C., Sánchez, E., Cuadrado, A. 1998. Identificación, delimitación y análisis de los elementos del paisaje necesarios para mejorar la coherencia de la Red Natura 2000. Región Alpina Española. Directiva Hábitat 92/43/CEE. Asesores Técnicos de Medio Ambiente (ATECMA) S.L., Madrid.

De Montis, A., Martín, B., Ortega, E., Ledda, A., Serra, V. 2017. Landscape fragmentation in Mediterranean Europe: A comparative approach. Land Use Policy 64, 83-94. http://doi. org/10.1016/j.landusepol.2017.02.028.

Estreguil, C., Caudullo, G., de Rigo, D., San Miguel, J. 2012. Forest Landscape in Europe: Pattern, Fragmentation and Connectivity. European Comission. http://doi.org/10.2788/77842.

EUROPARC-España. 2009. Conectividad ecológica y áreas protegidas. Herramientas y casos prácticos. Ed. FUNGOBE, Madrid, 86 pp.

Forman, R.T.T. 1995. Land mosaic. The ecology of landscapes and regions. Cambridge University Press, London.

Forman, R.T.T., Godron, M. 1986. Landscape Ecology. Wiley and Sons, Nueva York. http://doi. org/10.1002/9780470057339.

Forman, R.T.T., Sperling, D., Bissonette, J.A., Clevenger, A.P., Cutshall, C.D., Dale, V.H. 2003. Road ecology: science and solutions. Islands Press, Washington. http://doi.org/10.1002/ ep.670220307.

García, D. 2011. Efectos biológicos de la fragmentación de hábitats: nuevas aproximaciones para resolver un viejo problema. Ecosistemas 20 (2-3), 1-10.

Geneletti, D. 2004. Using spatial indicators and value functions to assess ecosystem fragmentation caused by linear infrastructures. International Journal of Applied Earth Observation and Geoinformation 5 (1), 1-15.

Giménez, J., Barón, A., Comas, M., González, C., Garau, J., Beidas, O., Oliver, M., Nadal, F.J. 2014. Hidrogeologia de les Illes Balears: Masses d'aigua càrstiques. Endins 36, 9-26.

Gurrutxaga, M.Lozano, P.J. 2006. Efectos de la fragmentación de hábitats y pérdida de conectividad ecológica dentro de la dinámica territorial. Polígonos. Revista de Geografia 16, 35-54. http:// doi.org/10.18002/pol.v0i16.410. 
Gurrutxaga, M., Lozano, P.J. 2010. Causas de los procesos territoriales de fragmentación de hábitats. Lurralde 33, 147-157.

Hargis, C.D, Bissonette, J.A, Turnes, D.L. 1999. The influence of forest fragmentation and landscape pattern on American martens. Ecological Application 36, 157-172. http://doi. org/10.1046/j.1365-2664.1999.00377.x.

Jaeger, J., Madriñán, L., Soukup, T., Schwick, C., Kienast, F. 2011. Landscape fragmentation in Europe. European Enviromental Agency-Swiss Federal Office for the Environment, Copenhagen, $87 \mathrm{pp}$.

Lindenmayer, D.B., Fischer, J. 2006. Habitat fragmentation and landscape change: An ecological and conservation synthesis. Island Press, Washington, $328 \mathrm{pp}$.

Llorens, L., Gil, L., Tébar, F.J. 2007. La vegetació de l'illa de Mallorca. Bases per a la interpretació $i$ gestió d'hàbitats. Govern de les Illes Balears, Conselleria de Medi Ambient, Palma de Mallorca, $261 \mathrm{pp}$.

Lorenzo-Lacruz, J., Morán-Tejeda, E. 2016. Spatio-temporal patterns of meteorological droughts in the Balearic Islands (Spain). Cuadernos de Investigación Geográfica 42 (1), 49-66. http:// doi.org/10.18172/cig.2948.

MacArthur R.H., Wilson E.O. 1963. An equilibrium Theory of Insular Zoogeography. Society for the study of evolution, Vol.17. http://doi.org/10.2307/2407089.

MacArthur R.H., Wilson E.O. 1967. The theory of island biogeography. Princeton University Press, Princeton, New Jersey, USA. http://doi.org/10.1515/9781400881376.

Mader, H.J. 1984. Animal habitat isolation by roads in agricultural fields. Biological conservation 29, 81-96. http://doi.org/10.1016/0006-3207(84)90015-6.

Mallarach, J.M., Marull, J. 2006. Impact assessment of ecological connectivity at the regional level: recent developments in the Barcelona Metropolitan Area. Impact Assessment and Project Appraisal 24 (2), 127-137. http://doi.org/10.3152/147154606781765228.

Martín, B., Mancebo, S., Ortega, A., López, E. 2006. Evaluación de los efectos del PEIT sobre la fragmentación de hábitats. In: III Congreso de Ingeniería Civil, Territorio y Medio Ambiente, Zaragoza.

Marín, B., Ortega, E., Mancebo, S., Otero, I. 2008. Fragmentación de los hábitats de la Red Natura 2000 afectados por el PEIT (Plan Estratégico de Infraestructuras y Transporte). Geofocus 8, 44-60.

Ministerio de Medio Ambiente y Medio Rural y Marino. 2010. Indicadores de fragmentación de hábitats causada por infraestructuras lineales de transporte. Documentos para la reducción de la fragmentación causada por infraestructuras de transporte, núm. 4. Ministerio de Medio Ambiente y Medio Rural y Marino, Madrid, $133 \mathrm{pp}$.

Moragues, L. 1993. Estudio geológic del sector meridional de les serres de Llevant (Mallorca). Bolletí Societat d'Història Naural de les Balears 36, 103-120.

Morera, C., Pintó, J., Romero, M. 2007. Paisaje, procesos de fragmentación y redes ecológicas: aproximación conceptual. In: O. Chassot, C. Morera (Eds.), Corredores biológicos: acercamiento conceptual y experiencia en America. Imprenta Nacional. San José, Costa Rica, pp. 11-47.

Múgica de la Guerra, M., de Lucio, J.V., Martínez, C., Sastre, P., Atauri-Mezquida J.A., Montes, C., Castro, H., Molina, F., García, M.R. 2002. Integración territorial de espacios naturales protegidos y conectividad ecológica en paisajes mediterráneos. Dirección General de la RENP y Servicios Ambientales, Consejería de Medio Ambiente, Junta de Andalucía, Sevilla.

Myers, N., Mittermeier, R.A., Mittermeier, C.G., Fonseca, G.A.B., Kent, J. 2000. Biodiversity hotspots for conservation priorities. Nature 403, 853-858. http://doi.org/10.1038/35002501.

Pearson, S.M., Turner, M.G., Gardner, R.H., O’Neill, R.V. 1996. An organism-based perspective of habitat fragmentation. In: R.C. Szaro, D.W. Johnston (Eds.) Biodiversity in managed landscapes. Theory and practice. Oxford University Press. 
Quine, C.P., Watts, K. 2009. Successful de-fragmentation of woodland by planting in an agricultural landscape? An assessment based on landscape indicators. Journal of Environmental Management 90 (1), 251-259. http://doi.org/10.1016/j.jenvman.2007.09.002.

Rita, J., Payeras, T. 2006. Biodiversidad de las plantas vasculares de las Islas Baleares. Orsis 21, 41-58.

Riitters, K.H., Wickham, J.D., Coulston, J.W. 2004 . A preliminary assessment of Montreal process indicators of forest fragmentation for the United States. Environmental Monitoring and Assessment 91 (1), 257-276. http://doi.org/10.1023/B:EMAS.0000009240.65355.92.

Robledo, P.A. 2005. Los Paleocolapsos kársticos en las plataformas carbonatadas del Mioceno superior de Mallorca: análisis geográfico, genético, geológico y evolutivo. Tesis Doctoral no publicada, Departamento de Ciencias de la Tierra. Universidad de las Islas Baleares.

Rodríguez, E., Gutiérrez, J. 2012. Análisis de vulnerabilidad de redes de carreteras mediante indicadores de accesibilidad y SIG: Intensidad y polarización de los efectos del cierre de tramos de la red de carreteras de Mallorca. Geofocus 12, 374-394.

Rosell C., Álvarez, G., Cahill, S., Campeny, R., Rodríguez, A., Séiler, C. 2003. Cost 341. La fragmentación del hábitat en relación con las infraestructuras de transporte en España. Ministerio de Medio Ambiente, Madrid.

Sánchez, J.A., Calvo, J.F. 1999. Raptor distribution in relation to landscape composition in semiarid Mediterranean habitats. Journal of Applied Ecology 36, 254-262. http://doi.org/10.1046/ j.1365-2664.1999.00396.x.

Santos, T., Tellería, J.L. 2006. Pérdida y fragmentación del hábitat: efecto sobre la conservación de las especies. Ecosistemas 15 (2), 3-12.

Serra, J., Bayer, X., López, M., Seguí, M. 2011. Les ratapinyades de les Illes Baleares: Distribució, avaluació i estat sanitari de les poblacions. Endins 35, 269-282.

Szek, M. 2012. Fragmentación del paisaje en áreas protegidas. Tesis Doctoral no publicada, Departamento de Geografía Física i Análisis Geográfica Regional. Universidad de Barcelona.

Unión Europea. 2010. Una Infraestructura verde. Comisión Europea.

Viada, C. 2006. Libro rojo de los vertebrados de las Baleares. Conselleria de Medi Ambient, $3^{\mathrm{a}}$ edición, $281 \mathrm{pp}$.

Vila, J., Varga, D., Llausàs, A., Ribas, A. 2006. Conceptos y métodos fundamentales en ecología del paisaje (landscape ecology). Una interpretación desde la geografía. Documents d'Anàlisi Geogràfica 48, 151-166. 